\title{
Transcriptomics reveal the molecular underpinnings of chemosensory proteins in Chlorops oryzae
}

\author{
Lin Qiu ${ }^{1 \dagger}$, Shunjie Tao ${ }^{1+}$, Hualiang He${ }^{1}$, Wenbing Ding ${ }^{1,2}$ and Youzhi $\mathrm{Li}^{1,2^{*}}$
}

\begin{abstract}
Background: Chemosensory proteins are a family of insect-specific chemical sensors that sense specific chemical cues and regulate insect behavior. Chemosensory proteins have been identified and analyzed in many insect species, such as Drosophila melanogaster, Bactrocera dorsalis and Calliphora stygia. This research has revealed that these proteins play a crucial role in insect orientation, predation and oviposition. However, little is known about the chemosensory proteins of Chlorops oryzae, a major pest of rice crops throughout Asia.

Results: Comparative transcription analysis of the genes of Chlorops oryzae larvae, pupae and adults identified a total of 104 chemosensory genes, including 25 odorant receptors (ORs), 26 odorant-binding proteins (OBPs), 19 ionotropic receptors (IRs), 23 gustatory receptors (GRs) and 11 sensory neuron membrane proteins (SNMPs). The sequences of these candidate chemosensory genes were confirmed and used to construct phylogenetic trees. Quantitative real-time PCR (qRT-PCR) confirmed that the expression of candidate OR genes in different developmental stages was consistent with the fragments per kilobase per million fragments (FPKM) values of differentially expressed genes (DEGs).
\end{abstract}

Conclusions: The identification of chemosensory genes in C. oryzae provides a foundation for the investigation of the function of chemosensory proteins in this species, which, in turn, could allow the development of new, improved methods of controlling this pest.

Keywords: Chlorops oryzae, Transcriptome, Chemosensory genes, Olfactory receptors, Expression profiling

\section{Background}

Olfactory and gustatory systems play crucial roles in insect orientation, oviposition, host-identification, mate choice and predator avoidance [1-14]. Chemoreception is mediated by odor and taste receptors which are responsible for identifying a diverse array of chemicals [15-17].

Chlorops oryzae (Diptera) cause significant economic damage to rice crops throughout Asia. Newly hatched larvae primarily burrow into the stem of rice plants and feed on the growing tips of developing leaves [18]. Most

\footnotetext{
* Correspondence: liyouzhi@hunau.edu.cn

${ }^{\dagger}$ Lin Qiu and Shunjie Tao contributed equally to this work.

${ }^{1}$ Hunan Provincial Key Laboratory for Biology and Control of Plant Diseases and Insect Pests, College of Plant Protection, Hunan Agricultural University, Changsha 410128, China

${ }^{2}$ Hunan Provincial Engineering \& Technology Research Center for

Biopesticide and Formulation Processing, Changsha 410128, China
}

recent studies have focused on the ecology and physiology of this species [18-20], and there is consequently relatively little information on its genetics. In this paper we present the results of genetic and phylogenetic analyses of putative chemosensory genes in C. oryzae.

The mechanism responsible for discriminating chemical cues in lepidopteran larvae has been well established [21-26]. In short, hydrophobic volatile molecules are solubilized and ferried from the external environment to sensory neurons where the chemical signals they carry are converted into electric signals [27]. In insects the process of chemoreception involves olfactory receptors (ORs), gustatory receptors (GRs) and ionotropic receptors (IRs). In addition, odorant binding proteins (OBPs), sensory neuron membrane proteins (SNMPs) and odorant-degrading enzymes (ODEs) also play an important role in regulating host behavior $[1,17,28,29]$.

(c) The Author(s). 2018 Open Access This article is distributed under the terms of the Creative Commons Attribution 4.0 International License (http://creativecommons.org/licenses/by/4.0/), which permits unrestricted use, distribution, and 
Insect odor and taste receptor genes were first identified in Drosophila melanogaster [30,31] and subsequent research identified the corresponding genes in other Dipteran species, including Anopheles gambiae, Musca domestica, Bactrocera dorsalis, Calliphora stygia, Glossina morsitans morsitans, Mayetiola destructor, Episyrphus balteatus and Eupeodes corollae [31-36]. ORs and GRs were first regarded as G-protein-coupled receptors (GPCRs) that share a common 7-transmembrane protein. However, subsequent research suggests that these two chemosensory receptors are not homologous with mammalian $O R$ genes [37]. Insect GRs have been classified into sweet, bitter, and carbon dioxide receptors [38-42]. More recently, a new class of ionotropic receptor, a variant sub-family of ionotropic glutamate chemosensory receptor (iGluR), has been identified in Drosophila [43]. Insect OBPs are small hydrophilic proteins that ferry hydrophobic chemical cues to ORs across the sensilla lymph [44-46]. In the lepidoptera, OBPs are usually divided into general odorant-binding proteins (GOBPs) and pheromone-binding proteins (PBPs). GOBPs and PBPs are involved in recognizing and transporting host plant odorants and pheromones $[47,48]$. SNMPs are the transmembrane domain-containing proteins thought to be involved in pheromone and general odorant reception $[49,50]$.

The transcriptome approach has been a recent advance in investigating the mechanisms underlying chemosensory proteins in various insect taxa [51-54]. We used this approach to identify candidate chemosensory genes (25 ORs, 26 OBPs, 19 IRs, 23 GRs and 11 SNMPs) in $C$. oryzae larvae, pupae and adults. We then constructed phylogenetic trees to infer the putative functions of each gene, and used quantitative real-time RT-PCR (qPCR) to confirm the expression patterns of $O R$ genes in each developmental stage. The identification of putative chemosensory genes is an essential first step for both fully understanding the molecular basis of an insects' chemosensory system, and developing better pest management tools.

\section{Results}

\section{Analysis of the C. oryzae transcriptome}

An Illumina HiSeq platform and Trinity assembly was used to sequence $C$. oryzae larvae, pupae and adult transcriptomes. We obtained 50.64 million (Larvae-1), 50.90 million (Larvae-2) and 44.41 million (Larvae-3) raw-reads from larvae, 55.06 million (Pupae-1), 55.73million (Pupae-2) and 50.59 million (Pupae-3) raw-reads from pupae, and 57.64 million (Adult-1), 62.21 million (Adult-2) and 56.50 million (Adult-3) raw-reads from adults (Additional file 1: Table S1). Filtering obtained 49.97 million (Larvae-1), 50.31 million (Larvae-2), 43.86 million (Larvae-3), 54.36 million (Pupae-1), 54.86 million (Pupae-2), 49.35 million (Pupae-3), 56.12 million (Adult-1), 61.06 million (Adult-2) and 54.82 million
(Adult-3), clean-reads. The final transcript dataset contained 201, 810 unigenes with a mean length of $835 \mathrm{bp}$ and N50 length of $1,243 \mathrm{bp}$.

\section{Homology and gene ontology (GO) annotation}

A total of 68, 745 (34.1\%) unigenes showed significant similarity to known proteins in the NCBI non-redundant protein database when the cut-off of E-value was set to $10^{-5}$. E-value distributions suggested that the assembled sequences had $65.3 \%$ homology $\left(<1 \mathrm{e}^{-30}\right)$ with proteins in the $\mathrm{Nr}$ database. However, homology fell to $34.7 \%$ when E-values were between $1 \mathrm{e}^{-30}$ and $1 \mathrm{e}^{-5}$ (Additional file 2: Figure S1a). Similarity distributions showed that $79.3 \%$ of sequences had more than $60 \%$ similarity, and $20.7 \%$ of sequences had $18-59 \%$ similarity, to sequences in the $\mathrm{Nr}$ database (Additional file 2: Figure S1b). Species with the highest proportion of similar genes were $M$. domestica (17.9\%) followed by Ceratitis copitata (15.8\%), Bactrocera cucurbitae (14.8\%), B. dorsalis (13.7\%) and D. melanogaster (3.4\%). (Additional file 2: Figure S1c).

Gene ontology (GO) analysis was used to categorize annotated genes into functional groups. Most genes were categorized into the "Biological Process", "Cellular Process", "Metabolic Process" and "Single-organism Process", categories and "Cell" and "Cell Part" comprised the highest proportion of the "Cellular Component" category (Additional file 2: Figure S1d). "Binding" and "Catalytic Activity" were the most common subcategories in the "Molecular Function" category (Additional file 2: Figure S1d).

\section{Candidate odorant receptors}

We identified 25 candidate ORs in larval, pupal and adult transcriptomes and constructed a phylogenetic tree of the similarity between these and ORs from three other Dipteran species; D. melanogaster, Calliphora stygia and B. dorsalis (Fig. 1). This indicated that the highly-conserved $C$. oryzae co-receptor, Orco (Cluster-3781.62429), shares 88.1, 87.7, and 85.8\% identity with Orco in C. stygia, B. dorsalis and D. melanogaster OR83b, respectively. Cluster-3781.168642 and Cluster-13,424.1 belong to the same cluster and share $68.3 \%$ identity based on amino acid sequences. Similarly, 94.2\% homology was found between Cluster-16,651.0 and Cluster-9598.0, and 73.4\% between Cluster-18,499.0 and Cluster-18,899.0, which were clustered in different groups. Interestingly, even though Cluster-3781.138000 and Cluster-13,269.0 were placed on the same branch they only share $44.0 \%$ identity. The remaining C. oryzae ORs were placed in different clusters, which is consistent with their highly divergent amino acid sequences. Cluster-10,102.1 was placed on a separate branch of the phylogenetic tree (Fig. 1). 


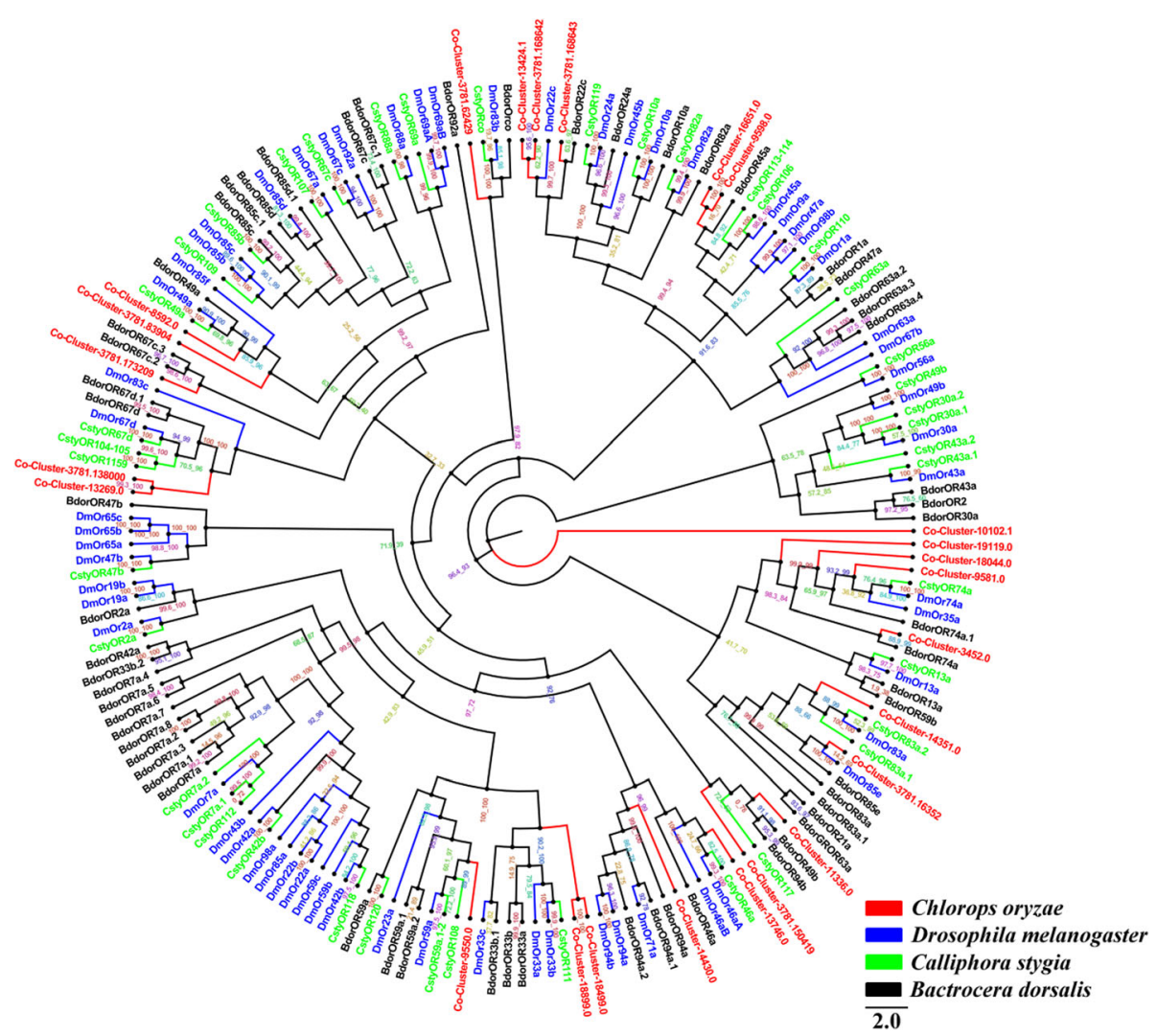

Fig. 1 Phylogenetic tree of relationships between Chlorops oryzae odorant receptors (ORs) and those of other species; B. dorsalis (Bdor, black), C. stygia (Csty, green) and D. melanogaster (Dm, blue) (Additional file 4). Bootstrap values after 1000 replications

\section{Candidate gustatory receptors}

We identified 23 candidate gustatory receptors (GR) from $C$. oryzae transcriptomes and constructed a phylogenetic tree of the relationships between these and $68 \mathrm{D}$. melanogaster GRs, 20 C. stygia GRs and 40 B. dorsalis GRs (Fig. 2). Cluster-10,678.0 and Cluster-6234.0 were placed on a single branch and share $12.7 \%$ identity. Cluster-8807.1 was classified with a D. melanogaster GR (Gr21a) (72.7\% identity). D. melanogaster Gr21a is a $\mathrm{CO}_{2}$ receptor in Drosophila [55]. Cluster-3781.37967 and Cluster-3781.170278 were grouped with another $D$. melanogaster $\mathrm{CO}_{2}$ receptor (Gr43a) (Fig. 2).

\section{Candidate ionotropic receptors}

We identified 19 candidate ionotropic receptor transcripts in C. oryzae. To distinguish putative IRs from iGluRs, all $C$. oryzae IRs were aligned with those from $D$. melanogaster, C. stygia and A. gambiae and a phylogenetic tree constructed of the resultant relationships. This placed Cluster-3781.106530 and Cluster-3781.15697 (49.9\% identity) on the same branch as the A. gambiae iGluRs (AgamGLURIIa, AgamGLURIIb, AgamGLURIIc, AgamGLURIId and AgamGLURIIe). Cluster-11,061.0 and Cluster-19,206.0 (97.1\% identity) were classified in single a group (Fig. 3). Cluster-3781.159004 and Cluster-3781.157618 were classified with the $D$. melanogaster ionotropic receptor superfamily and Co-Cluster-3781.174214 were classified with CstyIR107, CstyIR75d, DmelIR75d and CstyIR108-109. Cluster-8273.0 and Cluster-16,299.3 was classified separately from the IRs (iGluRs) of the other species. The remaining $C$. oryzae IRs were clustered on different branches, however, no $C$. oryzae IRs were placed on the IR7 branch (e.g. DmelIR7a, d, e, f, g; AgamIR7i, s, t, u, w, x). IRs on that branch may play various roles in insect olfaction.

\section{Candidate odorant binding proteins}

We identified 26 OBP transcripts from $C$. oryzae larvae, pupae and adults and constructed a phylogenetic tree of the relationships between these and OBPs from $D$. melanogaster and B. dorsalis. Cluster-3781.101742 and Cluster-3781.17418 were placed in a single group (47.3\% identity), Cluster3781.140399 and Cluster-3781.152546, which share 43.6\% identity, were placed on the same branch, as were 


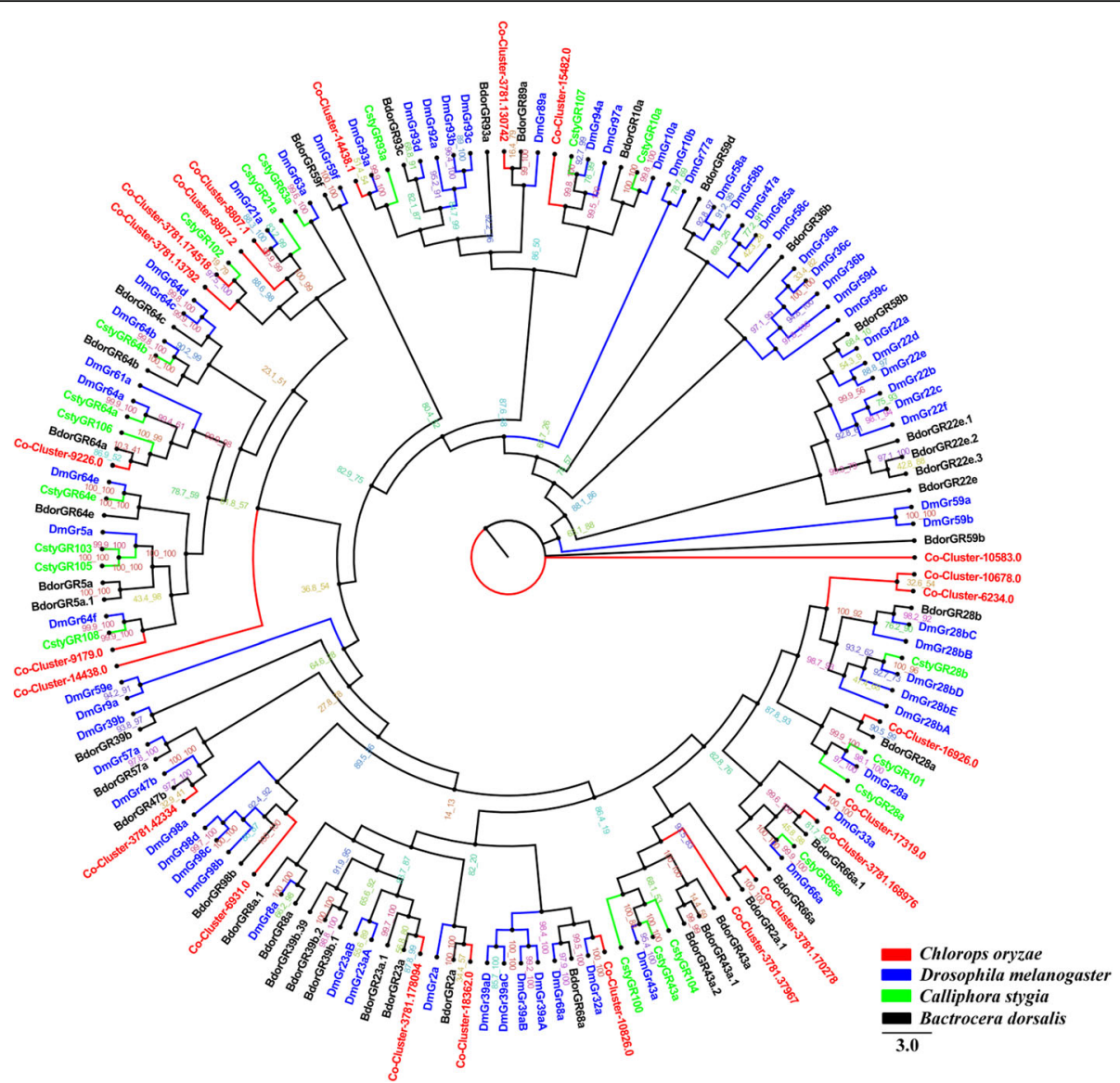

Fig. 2 Phylogenetic tree of relationships between Chlorops oryzae gustatory receptors (GRs) and those from other species; B. dorsalis (Bdor, black), C. stygia (Csty, green) and D. melanogaster (Dm, blue) (Additional file 5). Bootstrap values after 1000 replications

Cluster-3781.178870 and Cluster-3781.178841 (86.3\% identify) (Fig. 4). Four Co-OBPs (Cluster-3781.117642, Cluster3781.18626, Cluster-3781.36358 and Cluster-3781.38930) were clustered with BdorOBP-A5-1 and BdorOBP-A5-2. We found the orthologue of the BdorOBP-lush Cluster8053.0 in C. oryzae, these two OBPs were $56.2 \%$ identical at the amino acid level. Cluster-17,022.1 clustered separately with a large group of OBPs which suggests that it may have a novel function in odor recognition. The remaining Co-OBPs were placed in different clusters with those of the other species (Fig. 4).

\section{Candidate SNMPs}

Eleven $C$. oryzae SNMP transcripts were identified and aligned with those of two Dipteran species, D. melanogaster and $A$. gambiae. The resultant phylogenetic tree suggests that Cluster-3781.63294, Cluster-3781.49122, Cluster3781.47316 and Cluster-3781.160102 are similar to DmelSNMP1-2, AgamSNMP1-2 (Fig. 5). The remaining SNMPs were placed within a large SNMP superfamily.

\section{Differentially expressed genes (DEGs)}

The expression levels of chemosensory genes in larvae, pupae and adults were estimated as fragments per kilobase per million fragments (FPKM) values and the results shown in a heatmap (Fig. 6). Of the 25 ORs identified, 13 were more highly expressed in adults, including Orco (Cluster-3781.62429), and 10 were more highly expressed in pupae. Two ORs were more highly expressed in pupae compared to both larvae and adults (Fig. 6a). Eight OBPs were more highly expressed in adults and 7 were more highly expressed in pupae (Fig. 6b). Eight GRs were more highly expressed in adults, 8 were more highly expressed in pupae, and 6 were more highly expressed in larvae (Fig. 6c). Most IR genes were more highly expressed in pupae (Fig. 6d). Five SNMPS were more highly expressed in adults and 3 were more highly expressed in pupae (Fig. 6e).

\section{Specific expression profiles of candidate $O R$ genes in different developmental stages}

We used qRT-PCR to measure the expression levels of candidate $O R s$ in larvae, pupae and adults to confirm that the 


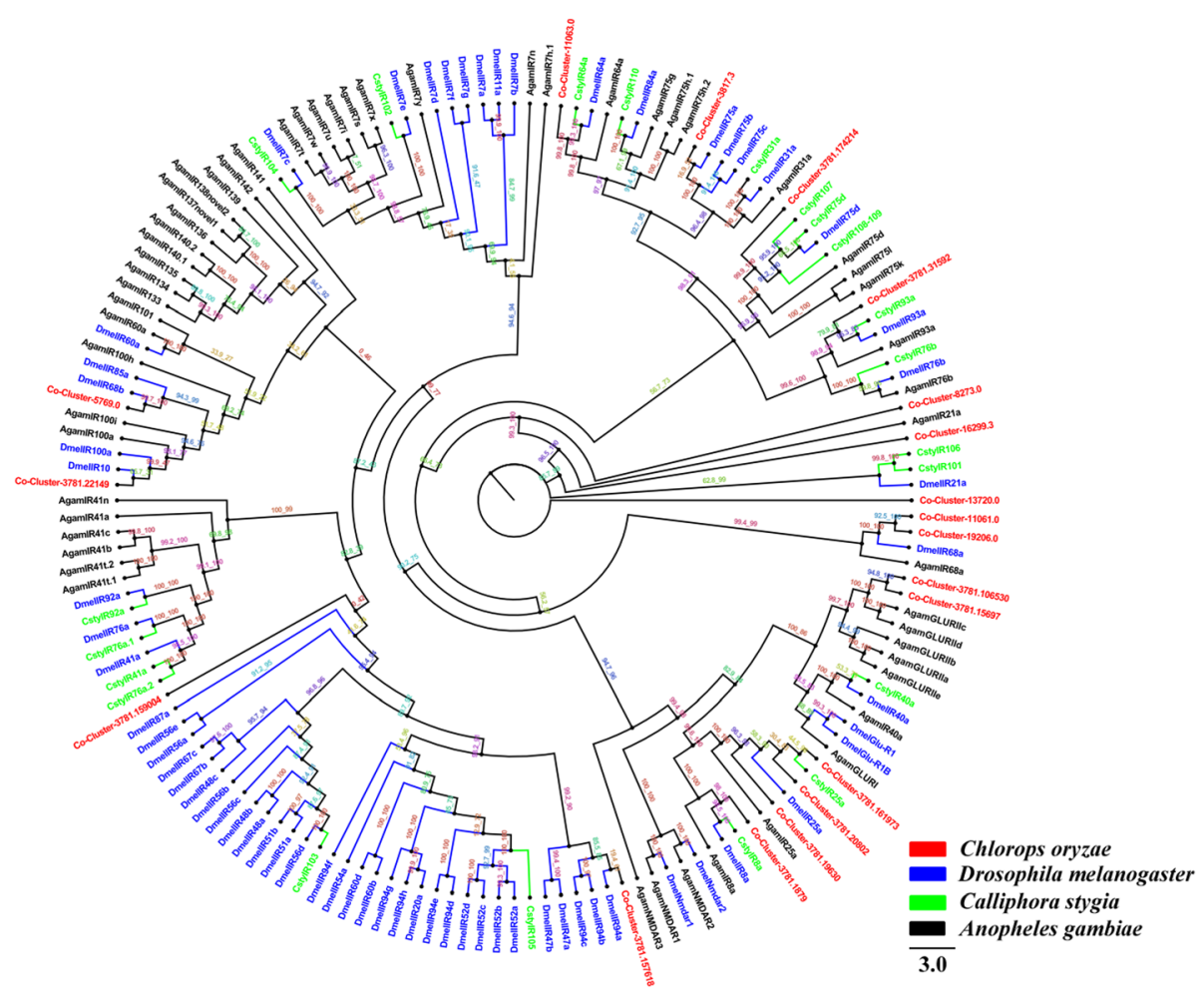

Fig. 3 Phylogenetic tree of relationships between Chlorops oryzae ionotropic receptors (IRs) and those of other species; A. gambiae (Agam, black), C. stygia (Csty, green) and D. melanogaster (Dmel, blue) (Additional file 6). Bootstrap values after 1000 replications

DEGs identified by comparative transcriptomic analysis are differentially expressed in these different developmental stages. We detected all 25 candidate ORs in the three developmental stages. The Orco gene was highly expressed in adults, as were Cluster-14,430.0, Cluster-8592.0 and Cluster9550.0 (Fig. 7). Cluster-3781.150419, Cluster-3781.16352, Cluster-14,351.0, Cluster-16,651.0 and Cluster-18,499.0, were more highly expressed in pupae compared to larvae and adults. These results are consistent with the results of the transcriptome analysis.

\section{Discussion}

C. oryzae is one of the most important insect pests of rice crops. In the majority of insect species, chemosensory proteins in the olfactory recognition system play a key role in foraging, orientation, mating and oviposition. To better understand how insects perceive olfactory chemical cues, we first identified candidate chemosensory proteins in transcriptomes of $C$. oryzae larvae, pupae and adults, then used qRT-PCR to investigate the expression profiles of $\mathrm{Co}$-ORs in these three different developmental stages. Our results provide new evidence of the molecular basis of olfactory proteins in chemosensory reception in $C$. oryzae that may help develop better methods of controlling this pest.
We used next generation sequencing technology to sequence transcriptomes from $C$. oryzae larvae, pupae and adults. De novo assembly of transcripts was performed using the Trinity method and a total of 68,745 unigenes were obtained from our sequence assembly, $34.1 \%$ of which showed similarities to known proteins in the NCBI non-redundant protein database. This percentage is less than that found for other Dipteran species $[35,54,56]$.

We identified 104 candidate chemosensory genes in $C$. oryzae. ORs, which connect binding proteins and olfactory sensory neurons to transduce olfactory signals, are the best known group of insect chemoreceptors. We identified $25 C$. oryzae ORs, less than those identified in C. stygia, G. morsitans morsitans, D. melanogaster, $M$. domestica and A. gambiae [32, 34-36]. The sequencing methods, or depth, used in these studies may have allowed the detection of genes with lower expression levels [57]. This suggests that different sample preparation and deep sequencing will be required to obtain more functional ORs from $C$. oryzae. Our phylogenetic analysis showed that Cluster-3781.138000 and Cluster-13,269 are part of the Dmor67d superfamily. Previous studies have found that OR67d is involved in the perception of the sex pheromone cis-vaccenyl acetate (cVA) [58-60]. Additional functional characterization of the candidate proteins we identified will provide 


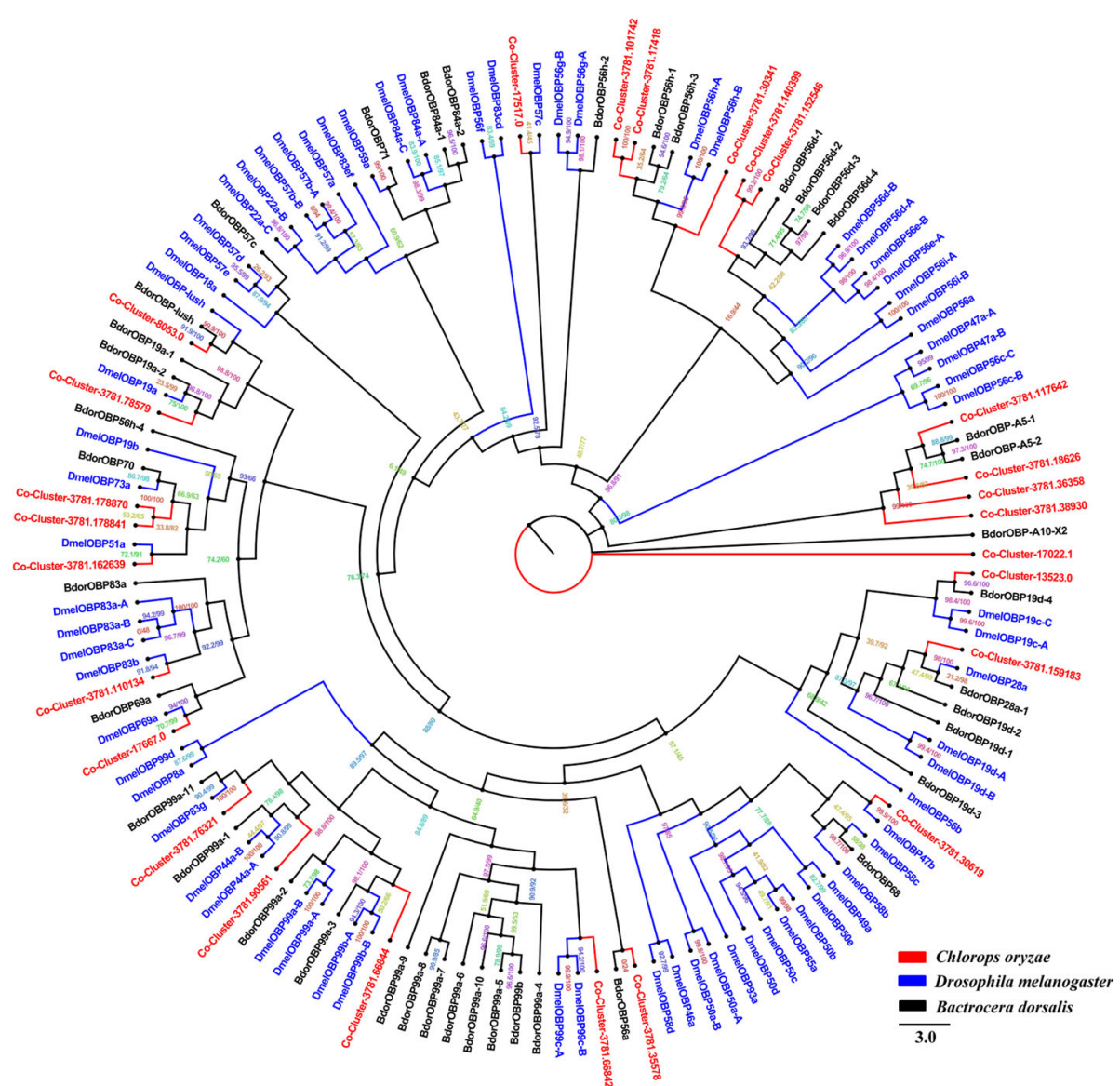

Fig. 4 Phylogenetic tree of relationships between Chlorops oryzae odorant binding proteins (OBPs) and those of other species; B. dorsalis (Bdor, black) and D. melanogaster (Dmel, blue) (Additional file 7). Bootstrap values after 1000 replications

further information on the pheromone reception mechanism in C. oryzae.

Our qRT-PCR results confirm that all $O R$ genes had different expression profiles in each development stage. Different olfactory organs may express different $O R$ genes in different developmental stages. For example, the maxillary palp in larvae plays the same vital role in host detection as the proboscis in adults [61-63].

OBPs also play a vital role or carrying odorants through the hemolymph to olfactory receptor neurons, and transducing the resultant signals to downstream effector molecules in the olfactory system [44]. We identified 26 OBPs in $C$. oryzae, fewer than have been found in other Dipteran species. This may reflect physiological and evolutionary differences between $C$. oryzae and the other Dipteran species [54, 64].

The function of OBPs in the Diptera is relatively well understood [65-69]. For example, DmelOBP-LUSH is involved in recognition of a $D$. melanogaster aggregation pheromone [65]. We found that the $C$. oryzae Cluster-8053.0 clustered with DmelOBP-LUSH and
BdorOBP-LUSH, however, because sequence specific attributes may affect OBP function and thereby influence behavior, additional research is required to confirm the function of OBPs in C. oryzae.

We identified 23 C. oryzae GRs, more than those found in the antennae of E. balteatus and E. corollae [54]. However, the number of GRs we found in C. oryzae is fewer than those reported in other Dipteran species, such as D. melanogaster [35]. GRs are known to function as taste and contact receptors [70], so it is not surprising that Cluster-9226.0 is homologous to the $D$. melanogaster sugar receptors $D m G r 61$ a and $D m G r 64$ a [71, 72]. Functional analysis indicates that GRs are involved in host-specific pollination behavior in some insects $[73,74]$. Similar functional analysis will be required to confirm the function of candidate GRs in C. oryzae.

IRs are conserved in the Diptera where they play a key role in the synaptic ligand gated ion channels involved in chemosensation. We identified 19 IRs in C. oryzae, fewer than have been reported in other Dipteran species $[35,54,75]$. In general, IRs function as chemoreceptors 


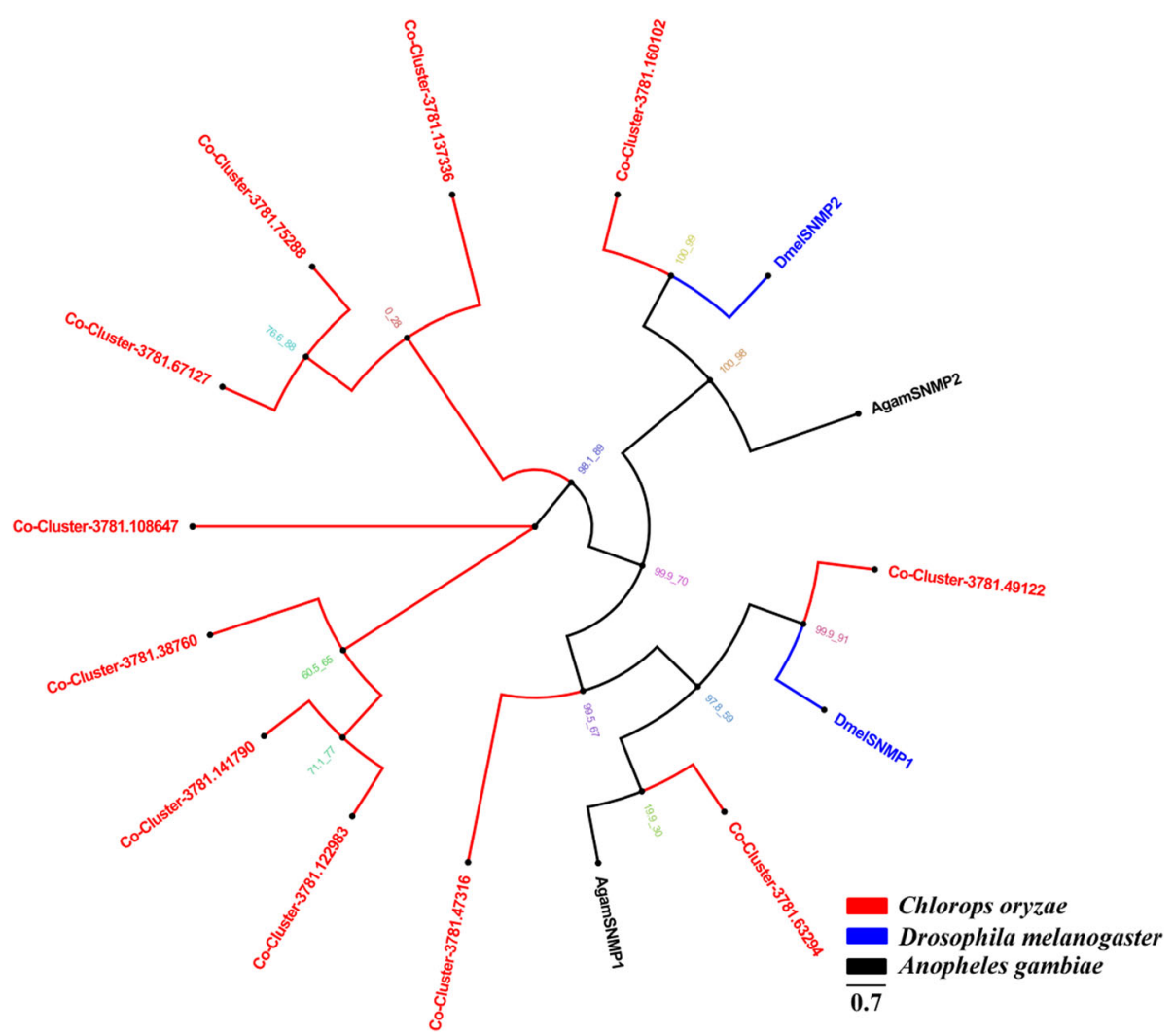

Fig. 5 Phylogenetic tree of relationships between Chlorops oryzae sensory neuron membrane proteins (SNMPs) and those of other species; $A$. gambiae (Agam, black) and D. melanogaster (Dmel, blue) (Additional file 8). Bootstrap values after 1000 replications

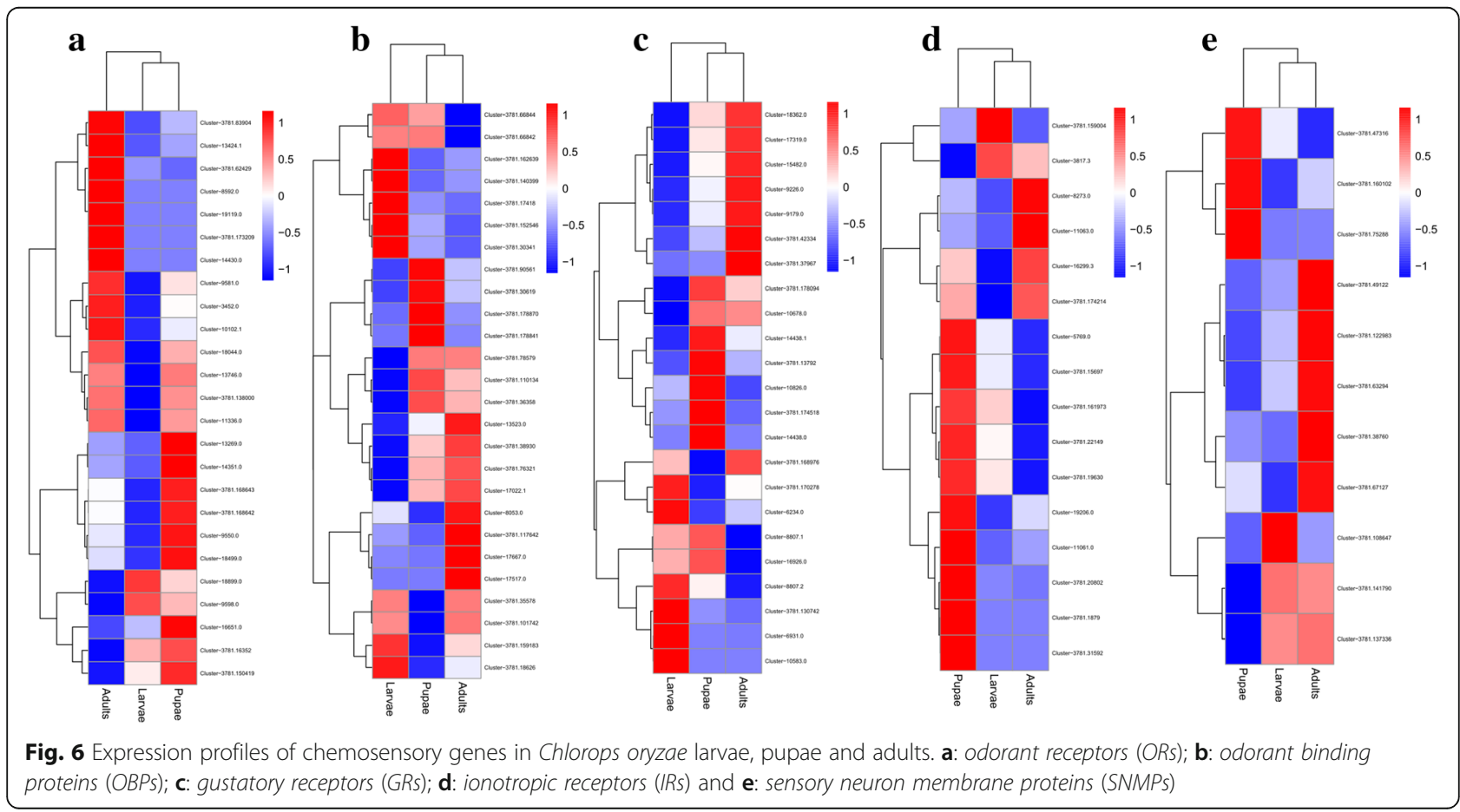




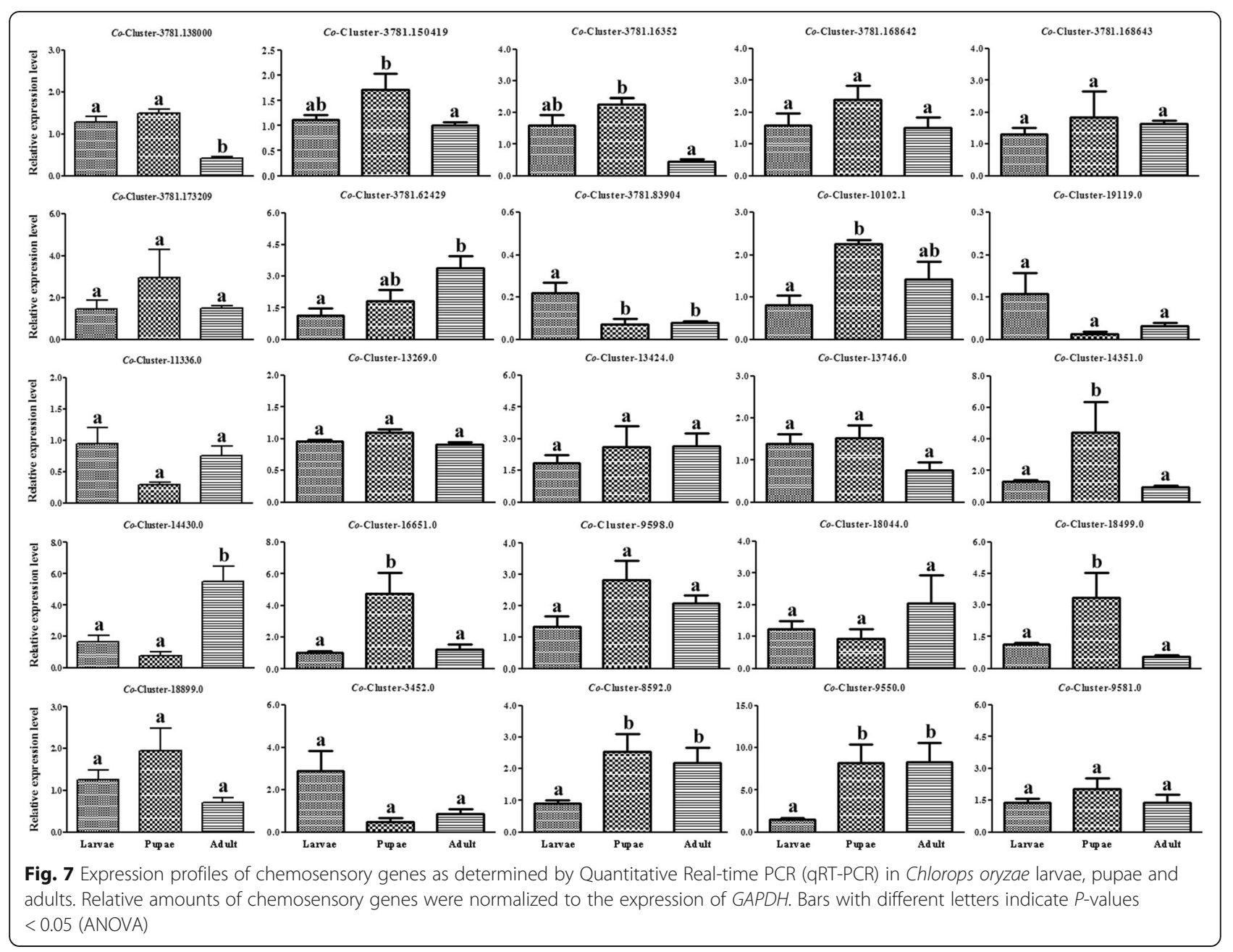

$[43,75]$ and are expressed in the peripheral and internal gustatory neurons associated with taste and food assessment [75], Further studies of $\mathrm{Co}$-IRs are required to reveal their physiological and ecological function. We also identified 11 C. oryzae SNMP transcripts. SNMPs are conserved throughout holometabolous insects and play important roles in pheromone detection [49, 50, 76-80]. Additional research is needed to verify how the putative SNMP proteins we identified mediate the behavior of $C$. oryzae.

\section{Conclusions}

The 104 candidate $C$. oryzae chemosensory proteins we identified comprise the first comprehensive list of chemosensory proteins in this important agricultural pest. Phylogenetic trees based on the sequence similarity of these putative proteins with similar proteins in other Dipteran species shed light on the molecular basis of olfactory and other behaviors in C. oryzae and provide a foundation for developing improved methods of controlling this pest.

\section{Methods}

Insect rearing and sample collection

C. oryzae larvae were collected in Hanshou County, Hunan province, China, in 2017, maintained in a laboratory and reared on fresh rice stems until pupation. Lab conditions were $28 \pm 1{ }^{\circ} \mathrm{C},>80 \%$ relative humidity, and a photoperiod of 16:8 (L:D) h. Samples of individual insects were collected from 2- to 5-day-old larvae, pupae and adults, respectively. All samples were immediately frozen in liquid nitrogen and stored at $-80^{\circ} \mathrm{C}$ until required.

\section{cDNA library construction and transcriptome analysis}

Total RNA of 30 larvae, 30 pupae and 30 adults were individually extracted using TRIzol reagent (Invitrogen, Carlsbad, CA, USA) according to the manufacturer's instructions. RNA integrity was verified with gel electrophoresis and concentration was measured with a Qubit ${ }^{\circ}$ RNA Assay Kit in Qubit ${ }^{\circ}$ 2.0 Flurometer (Life Technologies, CA, USA). $1.5 \mu \mathrm{g}$ of RNA per sample was used to construct the cDNA (Complementary DNA) library. 
Sequencing libraries were sequenced on an Illumina Hiseq platform and paired-end reads generated.

All RNAseq data were pre-processed through in-house perl scripts. Briefly, clean data (clean reads) were obtained by removing reads containing adapters, ploy- $\mathrm{N}$ and low-quality reads, from the raw data. The Trinity assembly was conducted based on the left.fq and right.fq using Trinity [81] with the min_kmer_cov set to 2 by default and all other parameters set to the default values. The raw sequence data has been uploaded to the National Center for Biotechnology Information (NCBI), under the accession number of SRR7528441 (C. oryzae Larvae-1), SRR7528446 (C. oryzae Larvae-2), SRR7528467 (C. oryzae Larvae-3), SRR7529086 (C. oryzae Pupae-1), SRR7529100 (C. oryzae Pupae-2), SRR7533623 (C. oryzae Pupae-3), SRR7534236 (C. oryzae Adult-1), SRR7534658 (Adult-2) and SRR7534603 (Adult-3).

The function of unigenes was inferred by aligning them against $\mathrm{Nr}$ (NCBI non-redundant protein sequences), Nt (NCBI non-redundant nucleotide sequences), Pfam (Protein family), KOG/COG (Clusters of Orthologous Groups of proteins), Swiss-Prot (A manually annotated and reviewed protein sequence database), KO (KEGG Ortholog database) and GO (Gene Ontology).

\section{Differential gene expression}

The differential expression of genes in larvae, pupae and adults was measured using the Fragments Per Kilobase of transcripts per Million mapped reads (FPKM) method [82], Differential expression in two conditions/groups (genes and samples) was measured using the DESeq $\mathrm{R}$ package (1.10.1). DESeq provides statistical routines for determining differential digital gene expression using a model based on the negative binomial distribution. The resulting $P$-values were adjusted using Benjamini and Hochberg's approach to control false discovery rate. Genes with an adjusted P-value $<0.05$ were considered to be differentially expressed.

\section{Identification of chemosensory genes}

Chemosensory receptor genes were verified by manually checking the amino acid sequences of all identified candidate receptors in BLASTX against the NCBI non-redundant protein database (e-value $<1 \mathrm{e}-5$ ) based on the identity and similarity to orthologous genes from other insect species. The Open reading frame (ORF) of candidate chemosensory genes was predicted by ORF finder tool (https://www.ncbi.nlm.nih.gov/orffinder/).

\section{Sequencing and phylogenetic analysis}

Amino acid sequence alignment was performed using the ClustalW method [83]. Phylogenetic trees of $C$. oryzae chemosensory genes were constructed in IQ-TREE using the best-fitting substitution-model with Maximum-likelihood [84]. Branch support was assessed by bootstrapping with 1000 replicates. OR sequences were obtained from $D$. melanogaster, $C$. stygia and $B$. dorsalis. The GR data set contained GR sequences identified in other Diptera, including D. melanogaster, C. stygia and B. dorsalis. The OBP data set contained OBP sequences from $D$. melanogaster and $B$. dorsalis. The IR data set contained IR sequences from $D$. melanogaster, C. stygia and A. gambiae. For the SNMP data set, we selected SNMP sequences from $D$. melanogaster and A. gambiae.

\section{Quantitative real-time PCR}

We used qRT-PCR with three replicates for each treatment to verify the expression of candidate $C$. oryzae chemosensory genes. cDNA was synthesized from total RNA using a PrimeScript RT reagent kit with gDNA eraser (perfect real time) (Takara, Dalian, China) according to the manufacturer's instructions. qRT-PCR primers were designed using the National Center for Biotechnology Information's profile server (https://www.ncbi.nlm.nih.gov/tools/primer-blast/) (Additional file 3: Table S2). The C. oryzae glyceraldehyde-phosphate dehydrogenase $(G A P D H)$ gene was used as the internal reference. A SYBR $^{\circ}$ Premix Ex Taq ${ }^{\text {Tm }}$ (TaKaRa, Dalian, China) and a Bio-rad Detection iQ2 System were used for PCR reactions as follows: $95^{\circ} \mathrm{C}$ for $30 \mathrm{~s}, 40$ cycles at $95^{\circ} \mathrm{C}$ for $10 \mathrm{~s}$, $59^{\circ} \mathrm{C}$ for $30 \mathrm{~s}$. Melting curve analysis was performed from $55^{\circ} \mathrm{C}$ to $95^{\circ} \mathrm{C}$ to determine the specificity of qPCR primers. To determine the efficiency of the qPCR primers, a standard curve (cDNA concentration vs. Ct) was produced with a 5 -fold dilution series of 3rd instar larvae cDNA corresponding to one microgram total RNA. qRT-PCR efficiencies were then calculated according to the equation: $E=\left(10^{[-1 / \text { slope }]}-\right.$ $1)^{*} 100[85,86]$. The $2^{-\Delta \Delta C t}$ method was used to analyze gene expression profiles [85]. Means and variances of treatments were analyzed with a one-way ANOVA implemented in the SPSS program for windows (SPSS, Chicago, IL, USA).

\section{Additional files}

Additional file 1: Table S1. Summary of the transcriptome sequencing data from the C. oryzae samples. (DOCX $15 \mathrm{~kb}$ )

Additional file 2: Figure S1. Results of BLASTx matches of Chlorops oryzae transcriptome unigenes and Gene ontology classification. a: E-values, b: gene identity, c: insect species in which homologous genes were matched. d: Gene ontology classifications of $C$. oryzae unigenes. (TIF $192 \mathrm{~kb}$ )

Additional file 3: Table S2. Primers of candidate ORs in C. oryzae used for qRT-PCR. (DOCX $16 \mathrm{~kb}$ )

Additional file 4: Protein sequences of ORs used to construct phylogenetic tree. (DOCX 52 kb) 
Additional file 5: Protein sequences of GRs used to construct phylogenetic tree. (DOCX $49 \mathrm{~kb}$ )

Additional file 6: Protein sequences of IRs used to construct phylogenetic tree. (DOCX $72 \mathrm{~kb})$

Additional file 7: Protein sequences of OBPs used to construct phylogenetic tree. (DOCX $28 \mathrm{~kb}$ )

Additional file 8: Protein sequences of SNMPs used to construct phylogenetic tree. (DOCX 17 kb)

\section{Abbreviations}

cDNA: Complementary DNA; DEGs: Differentially expressed genes; FPKM: Fragments per kilobase per million fragments; GO: Gene Ontology; GR: Gustatory receptor; IR: Ionotropic receptor; OBP: Odorant-binding protein; OR: Odorant receptor; ORF: Open reading frame; qRT-PCR: quantitative realtime polymerase chain reaction; SNMP: Sensory neuron membrane protein

\section{Acknowledgements}

We thank Weihua Ma (College of Plant Science and Technology, Huazhong Agricultural University) for technical assistance.

\section{Funding}

This project is supported by Hunan Provincial Department of Science and Technology Key R\&D Plan (2018NK2094).

\section{Availability of data and materials}

The authors declare that the data supporting the finding of this study are available in the article and its supplementary information files. The raw data submitted to the National Center for Biotechnology Information (NCBI) (https://www.ncbi.nlm.nih.gov/) with the accession numbers of SRR7528441, SRR7528446, SRR7528467, SRR7529086, SRR7529100, SRR7533623,

SRR7534236, SRR7534658 and SRR7534603

\section{Authors' contributions}

Performed the experiments: LQ, ST. Conceived and designed the experiments: $L Q, W D, H H, Y L$. Analyzed the data and wrote the manuscript: $L Q, Y L$. All authors read and approved the final manuscript.

\section{Ethics approval and consent to participate}

Not applicable.

\section{Consent for publication}

Not applicable.

\section{Competing interests}

The authors declare that they have no competing interests.

\section{Publisher's Note}

Springer Nature remains neutral with regard to jurisdictional claims in published maps and institutional affiliations.

Received: 19 July 2018 Accepted: 27 November 2018 Published online: 07 December 2018

\section{References}

1. de Bruyne M, Baker TC. Odor detection in insects: volatile codes. J Chem Ecol. 2008;34(7):882-97.

2. Francis F, Lognay $G$, Haubruge E. Olfactory responses to aphid and host plant volatile releases: (E)- $\beta$-Farnesene an effective Kairomone for the predator Adalia bipunctata. J Chem Ecol. 2004;30(4):741-55.

3. Verheggen FJ, Arnaud L, Bartram S, Gohy M, Haubruge E. Aphid and plant volatiles induce oviposition in an Aphidophagous hoverfly. J Chem Ecol. 2008;34(3):301-7.

4. Poppy GM. Tritrophic interactions: improving ecological understanding and biological control? Endvr. 1997;21(2):61-5.

5. Vet LEM, Dicke M. Ecology of Infochemical use by natural enemies in a Tritrophic context. Annu Rev Entomol. 1992;37(1):141-72.

6. Bargen H, Saudhof K, Poehling HM. Prey finding by larvae and adult females of Episyrphus balteatus. Entomol Exp Appl. 1998;87(3):245-54.
7. Sadeghi H, Gilbert F. Oviposition preferences of aphidophagous hoverflies Ecol Entomol. 2000;25(1):91-100.

8. Sadeghi H, Gilbert F. Aphid suitability and its relationship to oviposition preference in predatory hoverflies. J Anim Ecol. 2000;69(5):771-84.

9. Zhu J, Obrycki JJ, Ochieng SA, Baker TC, Pickett JA, Smiley D. Attraction of two lacewing species to volatiles produced by host plants and aphid prey. NW. 2005;92(6):277-81.

10. Van dGNW, Carlson JR. Insects as chemosensors of humans and crops. Nature. 2006;444(7117):302-7.

11. Almohamad R, Verheggen FJ, Francis F, Haubruge E. Predatory hoverflies select their oviposition site according to aphid host plant and aphid species. Entomol Exp Appl. 2007;125(1):13-21.

12. Verheggen FJ, Fagel Q, Heuskin S, Lognay G, Francis F, Haubruge E. Electrophysiological and behavioral responses of the multicolored Asian lady beetle, Harmonia axyridis pallas, to sesquiterpene semiochemicals. J Chem Ecol. 2007;33(11):2148-55.

13. Verheggen F, Ryne C, Olsson POC, Arnaud L, Lognay G, Högberg HE, Persson D, Haubruge E, Löfstedt C. Electrophysiological and behavioral activity of secondary metabolites in the confused flour beetle, Tribolium confusum. J Chem Ecol. 2007;33(3):525.

14. Carey AF, Carlson JR. Insect olfaction from model systems to disease control. Proc Natl Acad Sci U S A. 2011;108(32):12987-95.

15. Clyne PJ, Warr CG, Freeman MR, Lessing D, Kim J. Carlson JR. a novel family of divergent seven-transmembrane proteins: candidate odorant receptors in Drosophila. Neuron. 1999;22(2):327-38.

16. Vosshall LB, Amrein H, Morozov PS, Rzhetsky A, Axel R. A spatial map of olfactory receptor expression in the Drosophila antenna. Cell. 1999;96(5):725-36.

17. Leal WS. Odorant reception in insects: roles of receptors, binding proteins, and degrading enzymes. Annu Rev Entomol. 2013;58(1):373-91.

18. Takeda M. Genetic basis of photoperiodic control of summer and winter diapause in geographic ecotypes of the rice stem maggot, Chlorops oryzae. Entomol Exp Appl. 1998;86(1):59-70.

19. Takeda M, Nagata T. Photoperiodic responses during larval development and diapause of two geographic ecotypes of the rice stem maggot, Chlorops oryzae. Entomol Exp Appl. 1992;63(3):273-81.

20. Takeda M. Effects of photoperiod and temperature on larval development and summer diapause in two geographic ecotypes of the rice stem maggot, Chlorops oryzae Matsumura (Diptera: Chloropidae). Appl Entomol Zool. 1997;32(1):63-74.

21. Tanaka K, Uda Y, Ono Y, Nakagawa T, Suwa M, Yamaoka R, Touhara K. Highly selective tuning of a silkworm olfactory receptor to a key mulberry leaf volatile. Acta Agron Hung. 2009;19(11):881-90.

22. Krieger J, Gondesen I, Forstner M, Gohl T, Dewer Y, Breer H. HR11 and HR13 receptor-expressing neurons are housed together in pheromoneresponsive Sensilla Trichodea of male Heliothis virescens. Chem Senses. 2009;34(6):469-77.

23. Kakumani PK, Malhotra P, Mukherjee SK, Bhatnagar RK. A draft genome assembly of the army worm, Spodoptera frugiperda. Genomics. 2014; 104(2):134-43.

24. Hu P, Tao J, Cui M, Gao C, Lu P, Luo Y. Antennal transcriptome analysis and expression profiles of odorant binding proteins in Eogystia hippophaecolus (Lepidoptera: Cossidae). BMC Genomics. 2016;17(1):651.

25. He Y, Feng B, Guo Q. Du Y. age influences the olfactory profiles of the migratory oriental armyworm mythimna separate at the molecular level. BMC Genomics. 2017;18(1):32.

26. Zhang J, Wang B, Dong S, Cao D, Dong J, Walker WB, Liu Y, Wang G. Antennal transcriptome analysis and comparison of chemosensory gene families in two closely related noctuidae moths, Helicoverpa armigera and $\mathrm{H}$. assulta. PLoS One. 2015;10(2):e0117054.

27. Touhara K, Vosshall LB. Sensing odorants and pheromones with chemosensory receptors. Annu Rev Physiol. 2009;71(1):307-32.

28. Korsching S. Olfactory maps and odor images. Curr Opin Neurobiol. 2002; 12(4):387-92.

29. Rützler M, Zwiebel LJ. Molecular biology of insect olfaction: recent progress and conceptual models. J Comp Physiol A. 2005;191(9):777-90.

30. Suh E, Bohbot J, Zwiebel LJ. Peripheral olfactory signaling in insects. Curr Opin Insect Sci. 2014;6:86-92.

31. Andersson MN, Videvall E, Walden KK, Harris MO, Robertson HM, Löfstedt C. Sex- and tissue-specific profiles of chemosensory gene expression in a herbivorous gall-inducing fly (Diptera: Cecidomyiidae). BMC Genomics. 2014; 15(1):501. 
32. Robertson HM, Warr CG, Carlson JR. Molecular evolution of the insect chemoreceptor gene superfamily in Drosophila melanogaster. Proc Natl Acad Sci U S A. 2003;100(100 Suppl 2):14537-42.

33. Rinker DC, Zhou X, Pitts RJ, Rokas A, Zwiebel LJ. Antennal transcriptome profiles of anopheline mosquitoes reveal human host olfactory specialization in Anopheles gambiae. BMC Genomics. 2013;14(1):749.

34. Scott JG, Warren WC, Beukeboom LW, Bopp D, Clark AG, Giers SD, Hediger M, Jones AK, Kasai S, Leichter CA. Genome of the house fly, Musca domestica L., a global vector of diseases with adaptations to a septic environment. Genome Biol. 2014;15(10):466.

35. Leitch O, Papanicolaou A, Lennard C, Kirkbride KP, Anderson A. Chemosensory genes identified in the antennal transcriptome of the blowfly Calliphora stygia. BMC Genomics. 2015;16(1):255.

36. Obiero GFO, Mireji PO, Nyanjom SRG, Christoffels A, Robertson HM, Masiga DK. Odorant and gustatory receptors in the tsetse Fly Glossina morsitans morsitans. PLoS Negl Trop Dis. 2014;8(4):e2663.

37. Spehr M, Munger SD. Olfactory receptors. G protein-coupled receptors and beyond. J Neurochem. 2009;109(6):1570-83.

38. Freeman EG, Wisotsky Z, Dahanukar A. Detection of sweet tastants by a conserved group of insect gustatory receptors. Proc Natl Acad Sci U S A. 2014;111(4):1598.

39. Zhang HJ, Anderson AR, Trowell SC, Luo A, Xiang ZH, Correction XQY. Topological and functional characterization of an insect gustatory receptor. PLoS One. 2011;6(8):e24111.

40. Wei $X$, Zhang HJ, Alisha A. A sugar gustatory receptor identified from the foregut of cotton bollworm Helicoverpa armigera. J Chem Ecol. 2012;38(12): 1513-20.

41. Ni L, Bronk P, Chang EC, Lowell AM, Flam JO, Panzano VC, Theobald DL, Griffith LC, Garrity PA. A gustatory receptor paralog controls rapid warmth avoidance in Drosophila. Nature. 2013;500(7464):580-4.

42. Briscoe AD, Maciasmuñoz A, Kozak KM, Walters JR, Yuan F, Jamie GA, Martin SH, Dasmahapatra KK, Ferguson LC, Mallet J. Female behaviour drives expression and evolution of gustatory receptors in butterflies. PLoS Genet. 2013;9(7):e1003620.

43. Benton R, Vannice KS, Gomezdiaz C, Vosshall LB. Variant ionotropic glutamate receptors as chemosensory receptors in Drosophila. Cell. 2009; 136(1):149-62.

44. Hallem EA, Dahanukar A, Carlson JR. Insect odor and taste receptors. Annu Rev Entomol. 2006:51(10):113-35.

45. Wicher D, Schäfer R, Bauernfeind R, Stensmyr MC, Heller R, Heinemann SH, Hansson BS. Drosophila odorant receptors are both ligand-gated and cyclicnucleotide-activated cation channels. Nature. 2008;452(7190):1007.

46. Zwiebel $L J$, Takken W. Olfactory regulation of mosquito-host interactions. Insect Biochem Mol Biol. 2004;34(7):645-52.

47. Pelosi P, Maida R. Odorant-binding proteins in insects. Comp Biochem Physiol B Biochem Mol Biol. 1995;111(3):503.

48. Vogt RG. Biochemical diversity of odor detection: OBPs, ODEs and SNMPs. Insect pheromone biochemistry and molecular biology. San Diego: Academic Press; 2003. p. 391-45.

49. Jin $\mathrm{X}, \mathrm{Ha}$ TS, Smith DP. SNMP is a signaling component required for pheromone sensitivity in Drosophila. Proc Natl Acad Sci U S A. 2008;105(31): 10996-1001

50. Vogt RG, Miller NE, Litvack R, Fandino RA, Sparks J, Staples J, Friedman R, Dickens JC. The insect SNMP gene family. Insect Biochem Mol Biol. 2009; 39(7):448-56.

51. Liu Y, Gu S, Zhang Y, Guo Y, Wang G. Candidate olfaction genes identified within the Helicoverpa armigera antennal transcriptome. PLoS One. 2012; 7(10):e48260.

52. Cao D, Liu Y, Wei J, Liao X, Walker WB, Li J, Wang G. Identification of candidate olfactory genes in Chilo suppressalis by antennal transcriptome analysis. Int J Biol Sci. 2014;10(8):846

53. Hodges TK, Cosme LV, Athrey G, Pathikonda S, Takken W, Slotman MA Species-specific chemosensory gene expression in the olfactory organs of the malaria vector Anopheles gambiae. BMC Genomics. 2014;15(1):1089.

54. Wang B, Liu Y, Wang GR. Chemosensory genes in the antennal transcriptome of two syrphid species, Episyrphus balteatus and Eupeodes corollae (Diptera: Syrphidae). BMC Genomics. 2017;18(1):586

55. Kwon JY, Dahanukar A, Weiss LA, Carlson JR. The molecular basis of CO2 reception in Drosophila. Proc Natl Acad Sci U S A. 2007;104(9):3574-8.

56. Li XM, Zhu XY, Peng H, Lu X, Liang S, Li C, Wang ZQ, Deng DG, Zhang YN. Molecular characterization and sex distribution of chemosensory receptor gene family based on transcriptome analysis of Scaeva pyrastri. PLoS One. 2016;11(5):e0155323.

57. Li XM, Zhu XY, Wang ZQ, Wang Y, He P, Chen G, Sun L, Deng DG, Zhang YN. Candidate chemosensory genes identified in Colaphellus bowringi by antennal transcriptome analysis. BMC Genomics. 2015;16(1):1028.

58. Bartelt RJ, Schaner AM, Jackson LL. Cis-Vaccenyl acetate as an aggregation pheromone in Drosophila melanogaster. J Chem Ecol. 1985;11(12):1747-56.

59. Kurtovic A, Widmer A, Dickson BJ. A single class of olfactory neurons mediates behavioural responses to a Drosophila sex pheromone. Nature. 2007:446(7135):542.

60. Lebreton S, Trona F, Borreroecheverry F, Bilz F, Grabe V, Becher PG, Carlsson MA, Nässel DR, Hansson BS. Sachse S. feeding regulates sex pheromone attraction and courtship in Drosophila females. Sci Rep. 2015;5(3):13132.

61. Larsson MC, Domingos Al, Jones WD, Chiappe ME, Amrein H, Vosshall LB. Or83b encodes a broadly expressed odorant receptor essential for Drosophila olfaction. Neuron. 2004;43(5):703-14.

62. Kwon HW, Lu T, Rützler M, Zwiebel LJ. Olfactory responses in a gustatory organ of the malaria vector mosquito Anopheles gambiae. Proc Natl Acad Sci U S A. 2006;103(36):13526-31.

63. Sparks JT, Bohbot JD, Dickens JC. The genetics of chemoreception in the labella and tarsi of Aedes aegypti. Insect Biochem Mol Biol. 2014;48(3):8-16.

64. Xu PX, Zwiebel $\amalg$, Smith DP. Identification of a distinct family of genes encoding atypical odorant-binding proteins in the malaria vector mosquito, Anopheles gambiae. Insect Mol Biol. 2003;12(6):549-60.

65. Xu P, Atkinson R, Jones DN, Smith DP. Drosophila OBP LUSH is required for activity of pheromone-sensitive neurons. Neuron. 2005;45(2):193.

66. Matsuo T, Sugaya S, Yasukawa J, Aigaki T, Fuyama Y. Odorant-binding proteins OBP57d and OBP57e affect taste perception and host-plant preference in Drosophila sechellia. PLoS Biol. 2007;5(5):e118.

67. Biessmann H, Andronopoulou E, Biessmann MR, Douris V, Dimitratos SD, Eliopoulos E, Guerin PM, latrou K, Justice RW, Kröber T. The Anopheles gambiae odorant binding protein 1 (AgamOBP1) mediates indole recognition in the antennae of female mosquitoes. PLoS One. 2010;5(3):e9471.

68. Das S, Dimopoulos G. Molecular analysis of photic inhibition of bloodfeeding in Anopheles gambiae. BMC Physiol. 2008;8(1):23.

69. Pelletier J, Guidolin A, Syed Z, Cornel AJ, Leal WS. Knockdown of a mosquito odorant-binding protein involved in the sensitive detection of oviposition attractants. J Chem Ecol. 2010;36(3):245-8.

70. Clyne PJ, Warr CG, Carlson JR. Candidate taste receptors in Drosophila. Sci. 2000;287(5459):1830-4

71. Jiao Y, Moon SJ, Montell C. A Drosophila gustatory receptor required for the responses to sucrose, glucose, and maltose identified by mRNA tagging. Proc Natl Acad Sci U S A. 2007;104(35):14110-5.

72. Miyamoto T, Chen Y, Slone J, Amrein H. Identification of a Drosophila glucose receptor using $\mathrm{Ca}^{2+}$ imaging of single chemosensory neurons. PLoS One. 2013:8(1):e56304-25.

73. Jauker F, Diekötter T, Schwarzbach F, Wolters V. Pollinator dispersal in an agricultural matrix: opposing responses of wild bees and hoverflies to landscape structure and distance from main habitat. Landsc Ecol. 2009;24(4):547-55.

74. Raymond L, Plantegenest M, Vialatte A. Migration and dispersal may drive to high genetic variation and significant genetic mixing: the case of two agriculturally important, continental hoverflies (Episyrphus balteatus and Sphaerophoria scripta). Mol Ecol. 2013;22(21):5329-39.

75. Croset V, Rytz R, Cummins SF, Budd A, Brawand D, Kaessmann H, Gibson TJ, Benton R. Ancient protostome origin of chemosensory ionotropic glutamate receptors and the evolution of insect taste and olfaction. PLoS Genet. 2010;6(8):e1001064.

76. Jiang $X$, Pablo $P$, Ewald GW, Heinz B, Jürgen K. Identification and characterization of two "sensory neuron membrane proteins" (SNMPs) of the desert locust, Schistocerca gregaria (Orthoptera: Acrididae). J Insect Sci. 2016;16(1):33.

77. Benton R, Vannice KS, Vosshall LB. An essential role for a CD36-related receptor in pheromone detection in Drosophila. Nature. 2007;450(7167):289.

78. Rogers ME, Steinbrecht RA, Vogt RG. Expression of SNMP-1 in olfactory neurons and sensilla of male and female antennae of the silkmoth Antheraea polyphemus. Cell Tissue Res. 2001;303(3):433-46.

79. Rogers ME, Krieger J, Vogt RG. Antennal SNMPs (sensory neuron membrane proteins) of Lepidoptera define a unique family of invertebrate CD36-like proteins. J Neurobiol. 2001;49(1):47-61. 
80. Rogers ME, Sun M, Lerner MR, Vogt RG. Snmp-1, a novel membrane protein of olfactory neurons of the silk moth Antheraea polyphemus with homology to the CD36 family of membrane proteins. J Biol Chem. 1997:272(23):14792-9.

81. Grabherr MG, Haas BJ, Yassour M, Levin JZ, Thompson DA, Amit I, Adiconis $X$, Fan L, Raychowdhury R, Zeng Q. Full-length transcriptome assembly from RNA-Seq data without a reference genome. Nat Biotechnol. 2011;29(7):644.

82. Mortazavi A, Williams BA, Mccue $K$, Schaeffer L, Wold B. Mapping and quantifying mammalian transcriptomes by RNA-Seq. Nat Methods. 2008;5(7):621-8.

83. Larkin MA, Blackshields G, Brown NP, Chenna R, McGettigan PA, McWilliam $H$, Valentin F, Wallace IM, Wilm A, Lopez R. Clustal W and Clustal X version 2.0. Bioinformatics. 2007;23(21):2947-8.

84. Trifinopoulos J, Nguyen LT, Haeseler AV, Minh BQ. W-IQ-TREE: a fast online phylogenetic tool for maximum likelihood analysis. Nucleic Acids Res. 2016; 44(Web Server issue):W232-5.

85. Pfaffl MW. A new mathematical model for relative quantification in real-time RT-PCR. Nucleic Acids Res. 2001;29(9):e45.

86. Radonić A, Thulke S, Mackay IM, Landt O, Siegert W, Nitsche A. Guideline to reference gene selection for quantitative real-time PCR. Biochem Biophys Res Commun. 2004;313(4):856-62.

Ready to submit your research? Choose BMC and benefit from:

- fast, convenient online submission

- thorough peer review by experienced researchers in your field

- rapid publication on acceptance

- support for research data, including large and complex data types

- gold Open Access which fosters wider collaboration and increased citations

- maximum visibility for your research: over $100 \mathrm{M}$ website views per year

At $\mathrm{BMC}$, research is always in progress.

Learn more biomedcentral.com/submissions 